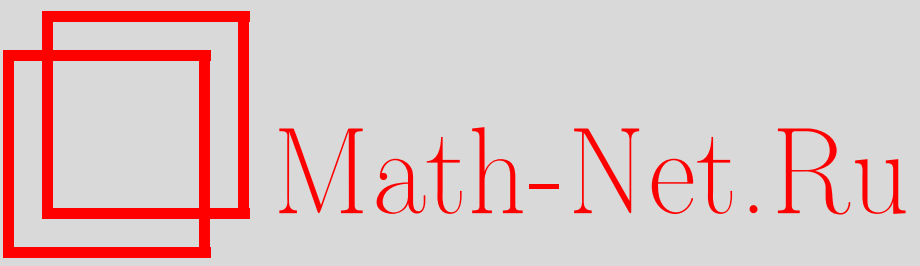

О. В. Висков, О формуле Швингера для скалярного произведения, УМH, 2005, том 60 , выпуск 2, 155-156

DOI: https://doi.org/10.4213/rm1412

Использование Общероссийского математического портала Math-Net.Ru подразумевает, что вы прочитали и согласны с пользовательским соглашением

http://www.mathnet.ru/rus/agreement

Параметры загрузки:

IP : 54.197 .217 .227

26 апреля 2023 г., $17: 29: 56$ 


\section{О ФОРМУЛЕ ШВИНГЕРА ДЛЯ СКАЛЯРНОГО ПРОИЗВЕДЕНИЯ}

О.В. Висков

Фиксируем натуралњное число $s$, и пусть $x=\left(x_{1}, x_{2}, \ldots, x_{s}\right)$ и $y=\left(y_{1}, y_{2}, \ldots, y_{s}\right)-$ два набора переменных, которые в дальнейшем будем рассматривать как векторы-строки. Для произвольной постоянной $(s \times s)$-матрицы $A=\left\|A_{i, j}\right\|$ обозначим (как это и сделано в [1])

$$
S(A, x, y)=x A y^{\top}=\sum_{i, j=1}^{s} x_{i} A_{i, j} y_{j},
$$

где символ ${ }^{\top}$ (здесь и в дальнейшем) означает операцию транспонирования.

Формула Швингера для скалярного произведения может быть записана в следующем виде (ср. с теоремой 4.1 в [1]):

Tеорема 1 (Schwinger's Master Theorem). Если произвольные постоянные $(s \times s)$-матриць $A$ и $B$ таковь, что матрица $I-A B$ обратима, то справедливо равенство

$$
\left.\exp \left\{S\left(A, \partial_{x}, \partial_{y}\right)\right\} \exp \left\{S\left(B^{\top}, x, y\right)\right\}\right|_{x=y=0}=\operatorname{det}(I-A B)^{-1},
$$

где $\partial_{x}=\left(\partial / \partial x_{1}, \partial / \partial x_{2}, \ldots, \partial / \partial x_{s}\right), \partial_{y}=\left(\partial / \partial y_{1}, \partial / \partial y_{2}, \ldots, \partial / \partial y_{s}\right)$ и символом I обозначена единичная матрица.

Справедливость равенства (1) для кососимметрических матриц $A$ и $B$ была установлена (без строгого доказательства) Швингером в отчете [2], опубликованном лишь много лет спустя в сборнике [3]. В [1] и [4] обсуждается связь соотношения (1) с имеющей фундаментальное значение в перечислительной комбинаторике Главной (Master) теоремой Макмагона [5] и с построением неэквивалентных неприводимых унитарных представлений общей унитарной группы.

Докажем, что справедливо следующее, обобщающее теорему 1 , утверждение:

Теорема 2. Если произвольные постоянные $(s \times s)$-матрицы $A$ и $B$ таковы, ито матрица $I-A B^{\top}$ обратима, то имеет место тожсдество

$$
\exp \left\{S\left(A, \partial_{x}, \partial_{y}\right)\right\} \exp \{S(B, x, y)\}=\operatorname{det}(C) \exp \{S(C B, x, y)\},
$$

где $C=\left(I-B A^{\top}\right)^{-1}$.

ДокАЗАТЕЛЬСтво. Введем параметр $t$ и обозначим

$$
u=\exp \left\{t S\left(A, \partial_{x}, \partial_{y}\right)\right\} \exp \{S(B, x, y)\} .
$$

Ясно, что введенная соотношением (3) функция $u$ является решением следующей задачи Коши:

$$
u_{t}=\sum_{i, j=1}^{s} A_{i, j} u_{x_{i} y_{j}},\left.\quad u\right|_{t=0}=\exp \{S(B, x, y)\} .
$$

Будем искать решение задачи (4) в виде

$$
u=\exp \{\alpha+S(\beta, x, y)\}
$$

где $\alpha$ и элементы $\beta_{i, j}(s \times s)$-матрицы $\beta=\left\|\beta_{i, j}\right\|$ - искомые функции параметра $t$.

Нетрудно проверить, что подстановка (5) в уравнение (4) приводит к равенствам

$$
\begin{gathered}
\beta_{m, n}^{\prime}=\sum_{i, j=1}^{s} \beta_{m, j} A_{i, j} \beta_{i, n},\left.\quad \beta_{m, n}\right|_{t=0}=B_{m, n}, \quad m, n=1,2, \ldots, s ; \\
\alpha^{\prime}=\sum_{i, j=1}^{s} \beta_{i, j} A_{i, j},\left.\quad \alpha\right|_{t=0}=0
\end{gathered}
$$


которые удобно переписать в матричной форме

$$
\begin{gathered}
\beta^{\prime}=\beta A^{\top} \beta,\left.\quad \beta\right|_{t=0}=B, \\
\alpha^{\prime}=\operatorname{Tr}\left(\beta A^{\top}\right),\left.\quad \alpha\right|_{t=0}=0 .
\end{gathered}
$$

В (7) (как и далее) символом $\operatorname{Tr}(\cdot)$ обозначен след матрицы $(\cdot)$.

Поскольку, как нетрудно убедиться, решение задачи (6) (по крайней мере при малых $t$ ) определяется соотношением

$$
\beta=\left(I-B A^{\top} t\right)^{-1} B,
$$

то интегрирование уравнения (7) с учетом (8) приводит к равенству

$$
\alpha=\operatorname{Tr}\left\{-\ln \left(I-B A^{\top} t\right)\right\} .
$$

Из равенства (9) и тождества Якоби, утверждающего, что для любой матрицы ( · )

$$
\operatorname{det} \exp (\cdot)=\exp \operatorname{Tr}(\cdot),
$$

видно, что

$$
\exp \alpha=\operatorname{det}\left(I-B A^{\top} t\right)^{-1} .
$$

Таким образом, решение задачи (4) имеет вид

$$
u=\operatorname{det}\left(I-B A^{\top} t\right)^{-1} \exp \left\{S\left(\left(I-B A^{\top} t\right)^{-1} B, x, y\right)\right\} .
$$

Для окончания доказательства теоремы остается лишь положить параметр $t$ равным единице.

В заключение отметим, что из теоремы 2 вытекают новые тождества для исползуемых в квантовой физике (см. [6], [7]) полиномов $L_{\alpha, \beta}(A)$, определяемых экспоненциалшной производящей функцией

$$
\sum_{m=0}^{\infty} \sum_{\alpha, \beta} x^{\alpha} L_{\alpha, \beta}(A) y^{\beta}=\exp \{S(A, x, y)\} .
$$

В этом соотношении использованы мултииндексные обозначения и внутреннее суммирование осуществляется по всем композициям $\alpha$ и $\beta$ числа $m$ в виде суммы $s$ неотрицательных целых чисел. Следует иметь в виду также тождество (см., например, равенство (4.4) в [1])

$$
\sum_{m=0}^{\infty} \sum_{\alpha, \beta} \alpha ! \beta ! L_{\alpha, \beta}(A) L_{\alpha, \beta}(B)=\operatorname{det}(I-A B)^{-1} .
$$

\section{СПИСОК ЛИТЕРАТУРЫ}

[1] W. Y. C. Chen, J. D. Louck // Adv. Math. 1998. V. 140. № 2. P. 207-236. [2] J. Schwinger. On angular momentum // U.S. Atomic Energy Commision Report NYO-3071, 1952. [3] L. C. Biedenharn, H. van Dam (Eds.). Quantum Theory of Angular Momentum. New York: Academic Press, 1965. [4] W. Y. C. Chen, H. W. Galbraith, J. D. Louck // Comput. Math. Appl. 2001. V. 41. № 9. P. 1199-1214. [5] I. P. Goulden, D. M. Jackson. Combinatorial Enumeration. New York: Wiley, 1983. [6] J. D. Louck // J. Math. Phys. 1965. V. 6. P. 1786-1804. [7] J. D. Louck // Amer. J. Phys. 1970. V. 38. P. 3-42.

Математический институт им. В. А. Стеклова РАН

E-mail: viskov@mi.ras.ru
Представлено Д. В. Аносовым Принято редколлегией 11.02 .2005 